\title{
Lévy Khinchin Formula on Commutative Hypercomplex System
}

\author{
Ahmed Moustfa Zabel \\ Department of Mathematics, Faculty of Science, Al-Azhar Univ., Nasr City, Cairo, \\ Egypt \\ e-mail : zabelahmed@hotmail.com \\ Buthinah Abdullateef Bin Dehaish \\ Department of Mathematics, Girls College of Education, Jeddah, Saudi Arabia \\ e-mail : math2000sa@yahoo.com
}

\begin{abstract}
A commutative hypercomplex system $L_{1}(Q, m)$ is, roughly speaking, a space which is defined by a structure measure $(c(A, B, r),(A, B \in \beta(Q))$. Such space has been studied by Berezanskii and Krein. Our main purpose is to establish a generalization of convolution semigroups and to discuss the role of the Lévy measure in the Lévy-Khinchin representation in terms of continuous negative definite functions on the dual hypercomplex system.
\end{abstract}

\section{Introduction}

The integral representation of negative definite functions is known in the literature as the Lévy-Khinchin formula. This was established for $G=R$ in the late 1930's by Lévy and Khinchin. It had been extended to Lie groups by Hunt [9] and by Parthasarathy et al [13] to locally compact abelian groups with a countable case. In 1969 Harzallah [7] gave a representation formula for an arbitrary locally compact abelian group. Hazod [8] obtained a Lévy-Khinchin formula for an arbitrary locally compact group. The general Lévy-Khinchin formula and the special case, where the involution is identical are due to Berg [4]. Lasser [12] deduced the Lévy-Khinchin formula for commutative hypergroups. Now these contribution may be viewed as a Lévy-Khinchin formula for negative definite functions defined on commutative hypercomplex systems.

Let $Q$ be a complete separable locally compact metric space of points $p, q, r \cdots, \beta(Q)$ be the $\sigma$-algebra of Borel subsets, and $\beta_{0}(Q)$ be the subring of $\beta(Q)$, which consists of sets with compact closure. We will consider the Borel measures; i.e. positive regular measures on $\beta(Q)$, finite on compact sets. The spaces of continuous functions of finite continuous function, of continuous functions vanish-

Received October 27, 2005.

2000 Mathematics Subject Classification: AMSC.43 XX.

Key words and phrases: Hypercomplex system, positive and negative definite functions, convolution semigroup, Lévy Khinchin formula. 
ing at infinity, and of continuous functions with compact support are denoted by $C(Q), C_{0}(Q), C_{\infty}(Q)$ and $C_{c}(Q)$, respectively. The space $C_{\infty}(Q)$ is a Banach space with norm

$$
\|.\|_{\infty}=\sup _{r \in Q}|(.)(r)| .
$$

Any continuous linear functional defined on the space $C_{0}(Q)$ with the inductive topology is called a (complex) Random measure. The space of Radon measures is denoted by $M(Q)$. Let $M_{b}(Q)=\left(C_{\infty}(Q)\right)^{\prime}$ be the Banach space of bounded Radon measures with norm

$$
\|\mu\|_{\infty}=\sup \left\{|\mu(f)|\left|f \in C_{\infty}(Q),\right| f \mid \leq 1\right\}
$$

and let $M_{c}(Q)$ be the space of Radon measures with compact support.

By $M_{1}(Q), M_{b}^{+}(Q)\left(M_{1}(Q) \subset M_{b}^{+}(Q)\right)$, we denote the set of Radon probability and bounded positive Radon measures on $Q$, respectively. The topology of simple convergence on functions from $C_{0}(Q)$ in the space of Radon measures, is called vague topology.

A hypercomplex system with the basis $Q$ is defined by its structure measure $c(A, B, r)(A, B \in \beta(Q) ; r \in Q)$. A structure measure $c(A, B, r)$ is a Borel measure in $A$ (respectively $B$ ) if we fix $B, r$ (respectively $A, r$ ) which satisfies the following properties:

(H1) $\forall A, B \in \beta_{0}(Q)$, the function $c(A, B, r) \in C_{0}(Q)$.

(H2) $\forall A, B \in \beta_{0}(Q)$ and $s, r \in Q$, the following associativity relation holds

$$
\int_{Q} c(A, B, r) d_{r} c\left(E_{r}, C, s\right)=\int_{Q} c(B, C, r) d_{r} c\left(A, E_{r}, s\right), \quad C \in \beta(Q)
$$

(H3) The structure measure is said to be commutative if

$$
c(A, B, r)=c(B, A, r), \quad\left(A, B \in \beta_{0}(Q)\right)
$$

A measure $m$ is said to be a multiplicative measure if

$$
\int_{Q} c(A, B, r) d m(r)=m(A) m(B) ; \quad A, B \in \beta_{0}(Q)
$$

(H4) We will suppose the existence of a multiplicative measure. Under certain relations imposed on the commutative structure measure, multiplicative measure exists. (See $[11]$ ).

For any $f, g \in L_{1}(Q, m)$, the convolution

$$
(f * g)(r)=\int_{Q} \int_{Q} f(p) g(q) d m_{r}(p, q)
$$


is well defined (See [2]).

The space $L_{1}(Q, m)$ with the convolution (1.1) is a Banach algebra which is commutative if (H3) holds. This Banach algebra is called the hypercomplex system with the basis $Q$.

A non zero measurable and bounded almost everywhere function $Q \ni r \rightarrow$ $\chi(r) \in C$ is said to be a character of the hypercomplex system $L_{1}$, if $\forall A, B \in$ $\beta_{0}(Q)$

$$
\begin{aligned}
& \int_{Q} c(A, B, r) \chi(r) d m(r)=\chi(A) \chi(B), \\
& \int_{C} \chi(r) d m(r)=\chi(C), \quad C \in \beta_{0}(Q) .
\end{aligned}
$$

(H5) A hypercomplex system is said to be normal, if there exists an involution homomorphism $Q \ni r \rightarrow r^{*} \in Q$, such that $m(A)=m\left(A^{*}\right)$, and $c(A, B, C)=$ $c\left(C, B^{*}, A\right), c(A, B, C)=c\left(A^{*}, C, B\right),\left(A, B \in \beta_{0}(Q)\right)$ where

$$
c(A, B, C)=\int_{C} c(A, B, r) d m(r)
$$

(H6) A normal hypercomplex system possesses a basis unity if there exists a point $e \in Q$ such that $e^{*}=e$ and

$$
c(A, B, e)=m\left(A^{*} \cap B\right), \quad A, B \in \beta(Q),
$$

we should remark that, for a normal hypercomplex system, the mapping

$$
L_{1}(Q, m) \ni f(r) \rightarrow f^{*}(r) \in L_{1}(Q, m)
$$

is an involution in the Banach algebra $L_{1}$, the multiplicative measure is unique and the characters of such a system are continuous (see [1]). A character $\chi$ of a normal hypercomplex system is said to be Hermitian if

$$
\chi\left(r^{*}\right)=\overline{\chi(r)} \quad(r \in Q) .
$$

Let $L_{1}(Q, m)$ be a hypercomplex system with a basis $Q$ and $\Phi$ a space of complex valued functions on $Q$. Assume that an operator valued function $Q \ni p \rightarrow R_{p}$ : $\Phi \rightarrow \Phi$ is given such that the function $g(p)=\left(R_{p} f\right)(q)$ belongs to $\Phi$ for any $f \in \Phi$ and any fixed $q \in Q$. The operators $R_{p}(p \in Q)$ are called generalized translation operators, provided that the following axioms are satisfied:

(T1) Associativity axiom: The equality

$$
\left(R_{p}^{q}\left(R_{q} f\right)\right)(r)=\left(R_{q}^{r}\left(R_{p} f\right)\right)(r)
$$

holds for any elements $p, q \in Q$. 
(T2) There exists an element $e \in Q$ such that $R_{e}$ is the identity in $\Phi$. See [3].

Clearly, the convolution (1.1) in the hypercomplex system $L_{1}(Q, m)$ and the corresponding family of generalized translation operators $R_{p}$ satisfy the relation

$$
(f * g)(p)=\int_{Q}\left(R_{s} f\right)(p) g\left(s^{*}\right) d s, \quad f, g \in L_{1}
$$

Denote by $\hat{Q}$ the support of the plancherel measure $\hat{m}[6]$. For any $M, N \in \beta(\hat{Q})$ and $\chi \in \hat{Q}$, we set

$$
\hat{c}(M, N, \chi)=\int_{Q} \overline{\chi(r)} \int_{M} \varphi(r) d \hat{m}(\varphi) \int_{N} \psi(r) d \hat{m}(\psi) d r, \quad \varphi, \psi \in \hat{Q} .
$$

Then, $\hat{c}(M, N, \chi)$ defines a structure measure on the dual hypercomplex system if and only if it belongs to $C_{0}(\hat{Q})$ for each fixed $M, N$ and the product of $\varphi, \psi \in \hat{Q}$ is a positive definite function.

The dual hypercomplex system $L_{1}(\hat{Q}, \hat{m})$ associated to $\hat{c}(M, N, \chi)$ will be constructed. The Plancherel measure $\hat{m}$ is a multiplicative measure for $\hat{c}(M, N, \chi)$. $L_{1}(\hat{Q}, \hat{m})$ is normal with the involution $\chi^{*}(r)=\overline{\chi(r)}$ and has a basis unity $\hat{e} \equiv 1(r)$. We denote by $\hat{\hat{m}}$ the Plancherel measure corresponding to the dual hypercomplex system and by $\hat{\hat{Q}}=\operatorname{supp} \hat{\hat{m}}$. We say that there is a duality if $Q=\hat{\hat{Q}}$. See [1].

\section{Negative definite functions}

Let $L_{1}(Q, m)$ be a commutative normal hypercomplex system with basis unity $e$.

Definition 2.1. A continuous bounded function $\psi: Q \rightarrow C$ is called negative definite if for any $r_{1}, \cdots, r_{n} \in Q$ and $c_{1}, \cdots, c_{n} \in C, n \in N$

$$
\sum_{i, j=1}^{n}\left[\psi\left(r_{i}\right)+\overline{\psi\left(r_{j}\right)}-\left(R_{r_{j}^{*}} \psi\right)\left(r_{i}\right)\right] c_{i} \overline{c_{j}} \geq 0
$$

By $P(Q)$ and $N(Q)$, we shall denote the set of all continuous positive definite functions and negative definite functions on $Q$ respectively. For example each constant $c \geq 0$ is a negative definite function. Obviously the following holds for a negative definite function $\psi$

$$
\psi(e) \geq 0, \overline{\psi(r)}=\psi\left(r^{*}\right),\left(R_{r^{*}} \psi\right)(r) \in R
$$

and

$$
\psi(r)+\psi\left(r^{*}\right) \geq R_{r^{*}} \psi(r)
$$

The following basic properties of negative definite functions on $Q$ are stated without proofs, for details and proofs, you can refer to [15].

Theorem 2.1. A function $\psi: Q \rightarrow C$ is negative definite if and only if the following conditions are satisfied: 
(i) $\psi(e) \geq 0, \psi$ is a continuous bounded function,

(ii) $\overline{\psi(r)}=\psi\left(r^{*}\right)$ for each $r \in Q$, and

(iii) for $r_{1}, \cdots, r_{n} \in Q$ and $c_{1}, \cdots, c_{n} \in C$ with $\sum_{i=1}^{n} c_{i}=0$, the summation

$$
\sum_{i, j=1}^{n}\left(R_{r_{j}^{*}} \psi\right)\left(r_{i}\right) c_{i} \overline{c_{j}} \leq 0
$$

Corollary 2.1. Let $\psi$ be a function on $Q$.

(i) If $\psi \in N(Q)$, then $r \mapsto \psi(r)-\psi(e)$ is negative definite.

(ii) If $\varphi \in P(Q)$, then $r \mapsto \varphi(e)-\varphi(r)$ is negative definite.

If the generalized translation operators $R_{t}$ extended to $L_{\infty}$ mapping $C_{0}(Q)$ into $C_{0}(Q \times Q)$, then inequality (2.1) is equivalent to the inequality

$$
\int_{Q} \int_{Q}\left(\psi(r)+\overline{\psi(s)}-\left(R_{s^{*}} \psi\right)(r)\right) x(r) \overline{x(s)} d r d s \geq 0, x \in L_{1}
$$

Theorem 2.2. Let $\psi: Q \rightarrow C$ be a continuous bounded function, $\psi(e) \geq 0$ and $\varphi_{t}: r \mapsto \exp (-t \psi(r))$ be positive definite for each $t \geq 0$. Then $\psi$ is negative definite.

Definition 2.2. A continuous function $h: Q \rightarrow R$ is called homomorphism if $h\left(r^{*}\right)=-h(r)$ and $\left(R_{r} h\right)(s)=h(r)+h(s), r, s \in Q$.

Lemma 2.1. If $h$ is a homomorphism, then $\psi=i$ is is negative definite.

Proof. Suppose $h$ is a homomorphism. Then for any $r_{1}, \cdots, r_{n} \in Q$ the matrix

$$
\left(\psi\left(r_{i}\right)+\overline{\psi\left(r_{j}\right)}-\left(R_{r_{s}^{*}} \psi\right)\left(r_{i}\right)\right)
$$

is the zero matrix, and it follows that $\psi \in N(Q)$.

Definition 2.3. A continuous function $q: Q \rightarrow R$ is called a quadratic form, if

$$
\left(R_{s} q\right)(r)+\left(R_{s^{*}} q\right)(r)=2(q(s)+q(r)), \quad r, s \in Q,
$$

By using (1.2) and (2.3), clearly a quadratic form $q$ satisfies:

$$
q(e)=0, \quad q\left(r^{*}\right)=q(r),
$$

and

$$
(\mu * \nu)(q)+(\mu * \bar{\nu})(q)=2(\mu(Q) \nu(q)+\nu(Q) \mu(q)), \mu, \nu \in M_{b}(Q)
$$


Lemma 2.2. Let $q$ be a quadratic form and $\mu \in M_{b}(Q)$. Then

$$
\mu^{2 n}(q)=4 n^{2} \mu(q)^{2 n-1} \mu(q)-n(2 n-1) \mu(Q)^{2 n-2} \mu * \bar{\mu}(q)
$$

for each $n \in N$, where $\mu^{n}$, the $n$-fold convolution of $\mu$.

Proof. (2.5) can be proved by induction. By (2.4), we obtain

$$
\mu^{2 n+2}(q)=2 \mu(Q)^{2 n+1} \mu(q)+2 \mu(Q) \mu^{2 n+1}(q)-\mu^{2 n+1} * \bar{\mu}(q)
$$

and

$$
\begin{aligned}
\mu^{2 n+1} * \bar{\mu}(q) & =\mu^{2 n} * \mu * \bar{\mu}(q) \\
& =\frac{1}{2}\left[\mu^{2 n} * \mu * \bar{\mu}(q)+\mu^{2 n} * \overline{\mu * \bar{\mu}(q)}\right] \\
& =\mu(Q)^{2 n} \mu * \bar{\mu}(q)+\mu(Q)^{2} \mu^{2 n}(q)
\end{aligned}
$$

and

$$
\begin{aligned}
\mu^{2 n+1}(q)= & \mu^{2 n} * \mu(q) \\
= & 2\left[\mu(Q)^{2 n} \mu(q)+\mu(Q) \mu^{2 n}(q)\right]-\mu^{2 n} * \bar{\mu}(q) \\
= & 2 \mu(Q)^{2 n} \mu(q)+2 \mu(Q) \mu^{2 n}(q) \\
& -\mu(Q)^{2 n-1} \mu * \bar{\mu}(q)-\mu(Q)^{2} \mu^{2 n-1}(q)
\end{aligned}
$$

Similar to $(2.8) \mu^{2 n}(q)=2 \mu(Q)^{2 n-1} \mu(q)$,

$$
\mu^{2 n}(q)=2 \mu(Q)^{2 n-1} \mu(q)+2 \mu(Q) \mu^{2 n-1}(q)-\mu(Q)^{2 n-2} \mu * \bar{\mu}(q)-\mu(Q)^{2} \mu^{2 n-2}(q)
$$

Then

$$
\begin{aligned}
\mu(Q) \mu^{2 n-1}(q)= & \frac{1}{2} \mu^{2 n}(q)-\mu(Q)^{2 n-1} \mu(q)+\frac{1}{2} \mu(Q)^{2 n-2} \mu * \bar{\mu}(q) \\
& +\frac{1}{2} \mu(Q)^{2} \mu^{2 n-2}(q)
\end{aligned}
$$

Substituting by (2.7) and (2.9) in (2.6), we get

$$
\mu^{2(n+1)}(q)=4(n+1)^{2} \mu(Q)^{2 n+1} \mu(q)-(n+1)(2 n+1) \mu(Q)^{2 n} \mu * \bar{\mu}(q) .
$$

Corollary 2.2. Let $q$ be a quadratic form. Then

$$
\lim _{n \rightarrow \infty} \frac{\left(R_{s}^{n} q\right)(s)}{4 n^{2}}=q(s)-\frac{1}{2}\left(R_{s^{*}} q\right)(s) .
$$

By using (2.4)and (1.2), the limit (2.10) can be proved easily. 
Let $\mathcal{S}$ be a smallest abelian semigroup containing $Q$ with unity $e$ and natural involution $s \mapsto s^{*}, s \in \mathcal{S}$.

A function $F: \mathcal{S} \rightarrow C$ will be called adapted if its restriction $f:=F \mid Q$ is locally bounded, measurable, and

$$
F(s * r)=\iint\left(R_{s} f\right)(r) d \mu(s) d \mu(r), \quad s, r \in Q
$$

Lemma 2.3. Let $\Psi: \mathcal{S} \rightarrow C$ be negative definite on $\mathcal{S}$ and

$$
\Psi(s * r)=\iint\left(R_{s} \psi\right)(r) d \mu(s) d \mu(r), \quad s, r \in Q
$$

Then $\psi$ is negative definite on $Q$.

Proof. Consider $s_{1}, \cdots, s_{n} \in \mathcal{S}$, then for $c_{1}, \cdots, c_{n} \in C$, we have

$$
\begin{aligned}
0 \leq & \sum_{i, j=1}^{n} c_{i} \overline{c_{j}}\left(\Psi\left(s_{i}\right)+\overline{\Psi\left(s_{j}\right)}-\Psi\left(s_{i} * s_{j}^{*}\right)\right) \\
= & \sum_{i, j=1}^{n} c_{i} \overline{c_{j}}\left(\iint \psi\left(s_{i}\right) d \mu\left(s_{j}\right) d \mu\left(s_{i}\right)+\iint \overline{\psi\left(s_{j}\right)} d \mu\left(s_{i}\right) d \mu\left(s_{j}\right)\right. \\
& -\iint\left(\left(R_{s_{i}} \psi\right)\left(s_{j}^{*}\right) d \mu\left(s_{j}\right) d \mu\left(s_{i}\right)\right) \\
= & \iint \sum c_{i} c_{j}\left(\Psi\left(s_{j}\right)+\overline{\Psi\left(s_{j}\right)}-\left(R_{s_{i}} \psi\right)\left(s_{j}^{*}\right)\right) d \mu\left(s_{i}\right) d \mu\left(s_{i}\right) .
\end{aligned}
$$

Then by $(2.2) \psi$ is negative definite.

Theorem 2.3. Nonnegative quadratic forms are negative definite.

Proof. Let $q: Q \rightarrow R^{+}$be a quadratic form, and for each $s, r \in \mathcal{S}$, put

$$
\mathbf{q}(s * r)=\iint\left(R_{s} q\right)(r) d \mu(s) d \mu(r) .
$$

Then

$$
\begin{aligned}
\mathbf{q}(s * r)+\mathbf{q}\left(s * r^{*}\right) & =\iint\left(\left(R_{s} q\right)(r)+\left(R_{s} q\right)\left(r^{*}\right)\right) d \mu(s) d \mu(r) \\
& =2 \iint\left(q(s)+q\left(r^{*}\right)\right) d \mu(s) d \mu(r) \\
& =2\left[\iint q(s) d \mu(s) d \mu(r)+\iint q(r) d \mu(s) d \mu(r)\right] \\
& =2[\mathbf{q}(s * e)+\mathbf{q}(r * e)]=2[\mathbf{q}(s)+\mathbf{q}(r)],
\end{aligned}
$$

which shows that $\mathbf{q}$ is a nonnegative quadratic form on $\mathcal{S}$. By [4] $q$ is negative definite and hence so is $q$ by Lemma 2.3 . 


\section{Covolution semigroups}

Let $L_{1}(Q, m)$ be a commutative normal hypercomplex system with basis $Q$ and basis unity $e$.

Definition 3.1. A family $\left(\mu_{t}\right)_{t>0}, \mu_{t} \in M_{b}^{+}(Q)$ is called a convolution semigroup on $Q$, if

(i) $\mu_{t}(Q) \leq 1$, for each $t>0$,

(ii) $\mu_{t_{1}} * \mu_{t_{2}}=\mu_{t_{1}+t_{2}}$ for $t_{1}, t_{2}>0$,

(iii) $\lim _{t \rightarrow 0} \mu_{t}=\varepsilon_{e}$

with respect to the vague topology on $M_{b}(Q)$.

Lemma 3.1. Let $\left(\mu_{t}\right)_{t>0}$ be a convolution semigroup on $Q$. Then, for $\chi \in \hat{Q}$, the function $t \mapsto \hat{\mu}_{t}(\chi), R^{+} \rightarrow C$ is continuous.

Proof. Using Urysohn's lemma, see [14], there exists $f \in C_{c}(Q)$ satisfing $0 \leq f \leq 1$ and $f(0)=1$. By (iii) and (i) above

$$
1=f(0)=\lim _{t \rightarrow 0}<\mu_{t}, f>\leq \lim _{t \rightarrow 0} \inf \mu_{t}(Q) \leq \lim _{t \rightarrow 0} \sup \mu_{t}(Q) \leq 1
$$

and this shows that

$$
\lim _{t \rightarrow 0} \mu_{t}=\varepsilon_{e} \quad \text { in the Bernolli topology }
$$

For $t_{1}, t_{0}>0$ and $\chi \in \hat{Q}$, we find, as in [5],

$$
\left|\hat{\mu}_{t}(\chi)-\hat{\mu}_{t_{0}}(\chi)\right| \leq\left|\hat{\mu}_{\left|t-t_{0}\right|}(\chi)-1\right|,
$$

and since the right-hand side, by (3.1), tends to zero uniformly on compact subsets of $\hat{Q}$, we get

$$
\lim _{t \rightarrow t_{0}} \mu_{t}=\mu_{t_{0}} \quad \text { in the Bernolli topology. }
$$

Theorem 3.1. Assume that $\hat{Q}$ is the dual of $Q$. If $\left(\mu_{t}\right)_{t>0}$ is a convolution semigroup on $Q$, then there exists exactly one negative definite function $\psi: \hat{Q} \rightarrow C$ with $R e \psi \geq 0$ such that

$$
\hat{\mu}_{t}(\chi)=\exp (-t \psi(\chi)) \text { for each } \chi \in \hat{Q}, t>0 .
$$

Proof. With some modifications to the proof of Theorem 8.3 in [5], we can prove this theorem easily.

Theorem 3.2. Assume that $Q$ has a duality and $\psi: \hat{Q} \rightarrow C$ is a negative definite 
function with $\operatorname{Re} \psi \geq 0$, such that $\hat{\mu}_{t}(\chi)=\exp (-t \psi(\chi))$ is positive definite for $t<0$. Then there exists a unique convolution semigroup $\left(\mu_{t}\right)_{t>0}$ on $Q$ such that $\psi$ is associated to $\left(\mu_{t}\right)_{t>0}$.

Proof. Since $\operatorname{Re} \psi \geq 0$, then $|\exp |(-t \psi(\chi) \mid \leq 1$. Thus by the duality of $Q$, there are unique determined measures $\mu_{t} \in M_{b}^{+}(Q), t>0$, such that $\hat{\mu}_{t}(\chi)=\exp (-t \psi(\chi))$. Obviously $\left(\mu_{t}\right)_{t>0}$ satisfies properties (i) and (ii). Further using the boundedness of $\psi$ on compact subsets of $\hat{Q}$,

$$
\lim _{t \rightarrow 0} \hat{\mu}_{t}(\chi)=\lim _{t \rightarrow 0} \exp (-t \psi(\chi))=1 .
$$

The duality of $Q$ defines a structure measure $\hat{c}$ as in (1.3) on the dual hypercomplex system $L_{1}(\hat{Q}, \hat{m})$. The Plancherel measure $\hat{m}$ is the multiplicative measure for $\hat{c}$.

Let $f \in C_{0}(Q), \varepsilon>0$, by [10], there exists $g \in C_{0}(\hat{Q})$, such that $\|f-\tilde{g}\|_{\infty}<\varepsilon$. Now we obtain

$$
\left|\mu_{t}(f)-\varepsilon_{e}(f)\right| \leq 2 \varepsilon+\int_{\hat{Q}}|g(\chi)|\left|\hat{\mu}_{t}(\bar{\chi})-1\right| d \hat{m}(\chi)
$$

which gives $\lim _{t \rightarrow 0} \mu_{t}=\varepsilon_{e}$ in the vague topology on $M_{b}(Q)$.

\section{The Lévy-Khinchin representation of the negative definite function}

Throughout this section, we consider $L_{1}(\hat{Q}, \hat{m})$ to be dual of $L_{1}(Q, m)$. Let $S$ denote the set of probability and symmetric measures on $\hat{Q}$ with compact support, i.e.

$$
S=\left\{\sigma \mid \sigma \in M_{1}^{+}(\hat{Q}) \cap M_{c}(\hat{Q}), \sigma(\chi)=\sigma(\bar{\chi})=\check{\sigma}(\chi)\right\}
$$

Lemma 4.1. Let $V$ be a compact neighbourhood of $e \in Q$. Then there exists a $\sigma \in S$ such that $\tilde{\sigma}(r) \leq \frac{1}{2}$ for each $r \in Q \backslash V$, where $\tilde{\sigma}$ is the Fourier transform of $\sigma$ on $\hat{Q}$.

Proof. By using Urysohn's lemma, there exists a function $\varphi \in C_{c}(Q)$ such that $0 \leq \varphi \leq 1, \varphi(r)=1$, for each $r \in V$ and $\operatorname{supp} \varphi \subset V$. Since $\varphi$ is a nonnegative constant function, then $\varphi \in P(Q)$. By Theorem 3.1 in [1] which is the analogue of Bochner's theorem for hypercomplex system, there is a positive bounded measure $\mu$ on $\hat{Q}$, such that $\tilde{\mu}=\varphi$. One can easily obtain that $\mu \in M_{1}(\hat{Q})$ and $\mu=\check{\mu}$. Choosing a compact symmetric set $J \subseteq \hat{Q}$ such that $\mu(J) \geq \frac{3}{4}$ and putting $\sigma=\mu(J)^{-1}(\mu \mid J)$, we find,

$$
\|\varphi-\tilde{\sigma}\|_{V}=\|\tilde{\mu}-\tilde{\sigma}\|_{V} \leq\|\mu-\sigma\|_{\infty} \leq \frac{1}{2}
$$

and thus $\tilde{\sigma}(r) \leq \frac{1}{2}$ for $r \in Q \backslash V$.

Theorem 4.1. Let $\left(\mu_{t}\right)$ be a convolution semigroup on $Q$ and $\psi: \hat{Q} \rightarrow C$ the 
negative definite function associated to $\left(\mu_{t}\right)_{t>0}$. Then the net $\left(\frac{1}{t} \mu_{t} \mid Q \backslash\{e\}\right)_{t>0}$ of positive measures on $Q \backslash\{e\}$ converges vaguely as $t \rightarrow 0$ to a measure $\mu$ on $Q \backslash\{e\}$. For every $\sigma \in S$, the function $\psi * \sigma-\psi$ is continuous positive definite on $\hat{Q}$ and the positive bounded measure $\mu_{\sigma}$ on $Q$ whose Fourier transform is $\psi * \sigma-\psi$ satisfies

$$
(1-\tilde{\sigma}) \mu=\mu_{\sigma} \mid Q \backslash\{e\}
$$

Proof. Let $\sigma \in S$. The measure $(1-\tilde{\sigma}) \frac{1}{t} \mu_{t}$ is positive bounded on $Q$, for $t>0$ and

$$
\begin{aligned}
{\left[(1-\tilde{\sigma}) \frac{1}{t} \mu_{t}\right]^{\wedge}(\chi) } & =\int(1-\tilde{\sigma}(r)) \frac{1}{t} \overline{\chi(r)} d \mu_{t}(r) \\
& =\frac{1}{t}\left[\int \overline{\chi(r)} d \mu_{t}(r)-\int \tilde{\sigma}(r) \overline{\chi(r)} d \mu_{t}(r)\right] \\
& =\frac{1}{t}\left[\hat{\mu}_{t}(\chi)-\iint \chi(s) \overline{\chi(r)} d \sigma(s) d \mu_{t}(r)\right] \\
& =\frac{1}{t}\left[\hat{\mu}_{t}(\chi)-\left(\hat{\mu}_{t} * \sigma\right)(\chi)\right] \\
& =\frac{1}{t}\left[1-\exp (-t \psi) *\left(\sigma-\varepsilon_{e}\right)\right](\chi) \text { for } \chi \in \hat{Q}
\end{aligned}
$$

Since $\lim _{t \rightarrow 0} \frac{1}{t}\left(1-e^{-t \psi}\right)=\psi$ uniformaly on compact subsets of $\hat{Q}$, we find that

$$
\lim _{t \rightarrow 0}\left[(1-\tilde{\sigma}) \frac{1}{t} \mu_{t}\right]^{\wedge}(\chi)=\left(\psi *\left(\sigma-\varepsilon_{e}\right)\right)(\chi)=\psi * \sigma(\chi)-\psi(\chi),
$$

pointwise (or uniformly over compact sets) on $\hat{Q}$. This shows that the function $\chi \mapsto \psi * \sigma(\chi)-\psi(\chi)$ is continuous positive definite, and furthermore, see ([5], 3.13) that

$$
\lim _{t \rightarrow 0}(1-\tilde{\sigma}) \frac{1}{t} \mu_{t}=\mu_{\sigma}
$$

in the Bernolli topology on $Q$, where $\mu_{\sigma}$ is positive bounded on $Q$ such that

$$
\hat{\mu}_{\sigma}=\psi * \sigma-\psi
$$

For, $\varphi \in C_{c}^{+}(Q)$ with supp $\varphi \subset Q \backslash\{e\}$, we may choose by Lemma 4.1, $\sigma \in S$ such that $\tilde{\sigma} \leq \frac{1}{2}$ in neighbourhood of $\operatorname{supp} \varphi$, let $\varphi^{\prime}$ be a function defined by

$$
r \stackrel{\varphi^{\prime}}{\mapsto} \begin{cases}\frac{\varphi(r)}{1-\tilde{\sigma}(r)} & \text { for } r \in \operatorname{supp} \varphi \\ 0 & \text { for } r \notin \operatorname{supp} \varphi\end{cases}
$$


this function belongs to $C_{c}^{+}(Q)$. Since

$$
\begin{aligned}
<\frac{1}{t} \mu_{t}, \varphi> & =\int \frac{1}{t} \mu_{t}(r) \varphi(r) d r \\
& =\int(1-\tilde{\sigma}(r)) \frac{1}{t} \mu_{t}(r) \frac{\varphi(r)}{1-\sigma(r)} d r \\
& =\left\langle(1-\tilde{\sigma}) \frac{1}{t} \mu_{t}, \varphi^{\prime}\right\rangle
\end{aligned}
$$

then

$$
\left.\lim _{t \rightarrow 0}\left\langle\frac{1}{t} \mu_{t}, \varphi\right\rangle=\lim _{t \rightarrow 0}\left\langle(1-\tilde{\sigma}) \frac{1}{t} \mu_{t}, \varphi^{\prime}\right\rangle=<\mu_{\sigma}, \varphi^{\prime}\right\rangle
$$

This shows, that there exists a positive measure $\mu$ on $Q \backslash\{e\}$ satisfies

$$
\mu=\lim _{t \rightarrow 0} \frac{1}{t} \mu_{t} \mid Q \backslash\{e\} \text { vaguely on } Q \backslash\{e\},
$$

and

$$
(1-\tilde{\sigma}) \mu=\mu_{\sigma} \mid Q \backslash\{e\} \quad \text { for } \sigma \in S .
$$

Definition 4.1. The positive measure $\mu$ on $Q \backslash\{e\}$ defined by Theorem 4.1 in (4.1) is called the Lévy measure for the convolution semigrpup $\left(\mu_{t}\right)_{t>0}$ on $Q$ (and also the Lévy measure for the negative definite function $\psi$ on $\hat{Q}$ ).

Theorem 4.2. Let $\mu$ denote the Lévy measure of a given convolution semigroup $\left(\mu_{t}\right)_{t>0}$. Then

(i) $\int_{Q \backslash\{e\}}(1-\operatorname{Re} \chi(r)) d \mu(r)<\infty$ for each $\chi \in \hat{Q}$.

(ii) If $V$ is a compact neighbourhood of e in $Q$, then $\mu \mid Q \backslash V \in M^{+}(Q)$

Proof. (i) For $\chi \in \hat{Q}$, let $\sigma=\frac{1}{2}\left(\varepsilon_{\chi}+\varepsilon_{\bar{\chi}}\right) \in S$; then $\tilde{\sigma}=\operatorname{Re} \chi(x)$ and by (4.1)

$$
\int_{Q \backslash\{e\}}(1-\operatorname{Re} \chi(r)) d \mu(r)=\int_{Q \backslash\{e\}}(1-\tilde{\sigma}(r)) d \mu(r)=\left.\mu_{\sigma}\right|_{Q \backslash\{e\}}<\infty
$$

The statement of (ii) follows as in ([5], 18.4).

The following two lemmas can be proved exactly as in ([5], 18.13 and 18.16).

Lemma 4.2. Let $h: \hat{Q} \rightarrow R$ be continuous and $h(1)=0 . h$ be a homomorphism if and only if $h * \sigma-h=0$ for each $\sigma \in S$.

Lemma 4.3. Let $q: \hat{Q} \rightarrow R$ be continuous with $q(\chi)=q(\bar{\chi}), q(1)=0 . q$ is a quadratic form if and only if $q * \sigma-q$ is a constant function for each $\sigma \in S$. 
Moreover $q$ is nonnegative if and only if $q * \sigma-q \geq 0$ for all $\sigma \in S$.

Corollary 4.1. Let $\left(\mu_{t}\right)$ be a convolution semigroup on $Q$. Assume that $\psi$ is the associated negative definite function. If the Lévy measure $\mu$ of $\left(\mu_{t}\right)_{t>0}$ is symmetric, then Im $\psi$ is a homomorphism. In particular iIm $\psi$ is negative definite. Further $\mu$ is also the Lévy measure of $\left(v_{t}\right)$, where $v_{t}=\mu_{t / 2} * \bar{\mu}_{t / 2}$.

Proof. $\check{\mu}=\mu$ is equivalent to $\check{\mu}_{\sigma}=\mu_{\sigma}$ for each $\sigma \in S$. This is equivalent to $\psi * \sigma-\psi$ being real valued for each $\sigma \in S$. Thus $\operatorname{Im} \psi * \sigma-\operatorname{Im} \psi=0$ for each $\sigma \in S$, and by Lemma $4.2 \operatorname{Im} \psi$ is a homomorphism. Thus $i \operatorname{Im} \psi$ is negative definite. Theorem 4.1 yields that $\left.\mu_{t}\right)_{t>0}$ and $\left(v_{t}\right)_{t>0}$ define the same class of measures $\mu_{\sigma}, \sigma \in S$. Therefore the uniqueness of the measure satisfing (4.1) implies the second assertian.

Since $Q$ is locally compact, then for every compact subset $k$ of $\hat{Q}$, there exists a constant $M_{k} \geq 0$, a neighbourhood $U_{k}$ of $e$ in $Q$ and a finite subset $N_{k}$ of $k$ such that for each $r \in U_{k}$

$$
\sup _{r}\{1-\operatorname{Re} \chi(r): \chi \in k\} \leq M_{k} \sup _{r}\left\{1-\operatorname{Re} \chi(r): \chi \in N_{k}\right\}
$$

see [13].

Lemma 4.4. Let $\mu$ be a positive symmetric measure on $Q \backslash\{e\}$ such that

$$
\int_{Q \backslash\{e\}}(1-\operatorname{Re} \chi(x)) d \mu(r)<\infty, \quad \text { for } \chi \in \hat{Q}
$$

The function $\psi_{\mu}: \hat{Q} \rightarrow R$ defined by

$$
\psi_{\mu}(\chi)=\int_{Q \backslash\{e\}}(1-\operatorname{Re} \chi(r)) d \mu(r) \quad \text { for } \chi \in \hat{Q}
$$

is continuous and negative definite.

Proof. Let $\chi_{0} \in \hat{Q}, \varepsilon>0$ and $K$ be a compact neighbourhood of $\chi_{0}$, then there exists a constant $M_{K} \geq 0$, a finite set $N_{K}=\left\{\chi_{1}, \cdots, \chi_{n}\right\} \subseteq \hat{Q}$ and a neighbourhood $U_{K}$ of $e$ in $Q$ such that

$$
\begin{aligned}
& \int_{U_{K} \backslash\{e\}} \sup _{\chi \in K}(1-\operatorname{Re} \chi(r)) d \mu(r) \\
\leq & M_{k} \int_{U_{k} \backslash\{e\}} \sup _{\chi \in N_{K}}(1-\operatorname{Re} \chi(r)) d \mu(r) \\
\leq & M_{k} \sum_{i=1}^{n} \int_{U_{k} \backslash\{e\}}\left(1-\operatorname{Re} \chi_{i}(r)\right) d \mu(r) \\
\leq & M_{k} \sum_{i=1}^{n} \int_{Q \backslash\{e\}}\left(1-\operatorname{Re} \chi_{i}(r)\right) d \mu(r)=M_{k} \sum_{i=1}^{n} \psi_{m}\left(\chi_{i}\right)
\end{aligned}
$$


Thus there exists a neighbourhood $V$ of $e$ such that

$$
\int_{V \backslash\{e\}}(1-\operatorname{Re} \chi(r)) d \mu(r)<\frac{\varepsilon}{4}
$$

for each $\chi \in K$. Since $\left.\mu\right|_{k \backslash v}$ is bounded, then there exists a neighbourhood $W \subseteq K$ of $\chi_{0}$ in $\hat{Q}$ such that

$$
\left|\int_{Q \backslash V}\left(\chi(r)-\chi_{0}(r)\right) d \mu(r)\right|<\frac{\varepsilon}{2}
$$

for each $\chi \in W$. For the continuity of $\psi_{\mu}$ we have

$$
\begin{aligned}
& \left|\psi_{\mu}(\chi)-\psi_{\mu}\left(\chi_{0}\right)\right| \\
= & \mid \int_{Q \backslash\{e\}}\left(1-\operatorname{Re} \chi(r) d \mu(r)-\int_{Q \backslash\{e\}}\left(1-\operatorname{Re} \chi_{0}(r) d \mu(r) \mid\right.\right. \\
= & \mid \int_{V \backslash\{e\}}\left(1-\operatorname{Re} \chi(r) d \mu(r)-\int_{V \backslash\{e\}}\left(1-\operatorname{Re} \chi_{0}(r) d \mu(r)+\int_{Q \backslash V}\left(\operatorname{Re} \chi_{0}\right)(r)-\operatorname{Re} \chi(r) d \mu(r) \mid\right.\right. \\
\leq & \int_{V \backslash\{e\}}\left(1-\operatorname{Re} \chi(r) d \mu(r)+\int_{V \backslash\{e\}}\left(1-\operatorname{Re} \chi_{0}(r) d \mu(r)+\left|\int_{Q \backslash V}\left(\operatorname{Re} \chi_{0}\right)(r)-\operatorname{Re} \chi(r) d \mu(r)\right|\right.\right. \\
< & \varepsilon
\end{aligned}
$$

for each $\chi \in W$. From (4.3) and (4.4), the continuity of $\psi_{\mu}$ is verified. In order to show that $\psi_{\mu}$ is negative definite, it is sufficient to prove that $\mu$ is a Lévy measure for it. For $f \in C_{c}^{+}(\hat{Q})$ such that $f(\bar{\chi})=f(\chi)$ and $\int f(\chi) d x=1$, we may apply Fubini's theorem to find

$$
\begin{aligned}
\left(\psi_{\mu} * f\right)(\chi) & =\int_{\hat{Q}}\left(R_{\eta} f\right)(\chi) \psi_{\mu}(\eta) d \eta \\
& =\int_{\hat{Q}} f(\eta) \int_{Q \backslash\{e\}}[1-\operatorname{Re} \chi(r) \eta(r)] d \mu(r) \\
& =\int_{Q \backslash\{e\}}[1-\operatorname{Re} \chi(r) \tilde{f}(r)] d \mu(r)
\end{aligned}
$$

In particular we have for $\chi=1$

$$
\int_{Q \backslash\{e\}}(1-\tilde{f}(r)) d \mu(r)=\int f(\eta) \psi_{\mu}(\eta) d \eta
$$

The measure $d \tau(r)=(1-\tilde{f}(r)) d \mu(r)$ is thus positive bounded on $Q \backslash\{e\}$. Then can be consider as a positive bounded measure on $Q$ and we have that

$$
\hat{\tau}(\chi)=\operatorname{Re} \hat{\tau}(\chi)=\int_{Q \backslash\{e\}} \operatorname{Re} \chi(r)(1-\tilde{f}(r)) d \mu(r) \quad \text { for } \chi \in \hat{Q} .
$$


Put $f=\sigma$ in (4.5). Then

$$
\begin{aligned}
\psi_{\mu} * \sigma(\chi)-\psi_{\mu}(\chi) & =\int_{Q \backslash\{e\}} \operatorname{Re} \chi(r)(1-\tilde{\sigma}(r)) d \mu(r) \\
& =\int_{Q \backslash\{e\}} \operatorname{Re} \chi(r)(1-\tilde{\sigma}(r)) d \mu(r)
\end{aligned}
$$

i.e. $\psi_{\mu} * \sigma-\psi_{\mu}$ is the Fourier transform of the measure $(1-\tilde{\sigma})(r) \mid \mu$ and by the result (4.1) of Theorem 4.1, the measure $\mu$ is Lévy measure of $\psi_{\mu}$.

Theorem 4.3. Let $\left(\mu_{t}\right)$ be a convolution semigroup on $Q$ with an associated negative definite function $\psi: \hat{Q} \rightarrow C$, and Lévy measure $\mu$. Assume that $\mu$ is positive symmetric on $Q \backslash\{e\}$ such that

$$
\int_{Q \backslash\{e\}}(1-\operatorname{Re} \chi(r) d \mu(r)<\infty \quad \text { for } \chi \in \hat{Q} .
$$

Then

$$
\psi(\chi)=C+i h(\chi)+q(\chi)+\int_{Q \backslash\{e\}}(1-\operatorname{Re} \chi(r)) d \mu(r)
$$

for $\chi \in \hat{Q}$, where $C$ is a nonnegative constant, $h: \hat{Q} \rightarrow R$, is a continuous homomorphism, $q: \hat{Q} \rightarrow R$, is a nonnegative quadratic form. Moreover $c, h, q$ in (4.6) are determined uniquely by $\left(\mu_{t}\right)_{t>0}$ such that $c=\psi(1), h=\operatorname{Im} \psi$, and

$$
q(\chi)=\lim _{n \rightarrow \infty}\left[\frac{\left(R_{\chi}^{n} \psi\right)(\chi)}{4 n^{2}}+\frac{\left(R_{\bar{\chi}}^{n} \psi\right)(\chi)}{2 n}\right]
$$

Proof. Since $\mu$ is symmetric, by corollary $4.1, h=\operatorname{Im} \psi$ is a homomorphism, and $i h \in N(\hat{Q})$. Let $C=\psi(1)$ then by Corollary 2.1 , the function $\psi-C I \in N(\hat{Q})$ with the Lévy measure $\mu$. Further the function $\psi^{\prime}=\psi-C I-i h \in N(\hat{Q})$ associated to the same Lévy measure $\mu$.

By Theorem 4.2, the function

$$
\psi_{\mu}(\chi)=\int_{Q \backslash\{e\}}(1-\operatorname{Re} \chi(r)) d \mu(r)
$$

is finite at all $\chi \in \hat{Q}$, and by Lemma 4.4, it follows that the function $q=\psi^{\prime}-\psi_{\mu}$ is continuous, real valued, symmetric and $q(1)=0$. Using Lemma 4.2, for $\sigma \in S$ we get

$$
\psi^{\prime} * \sigma-\psi^{\prime}=\psi * \sigma-\psi
$$

and one can easily obtain

$$
\psi_{\mu} * \sigma-\psi_{\mu}=\int_{Q\{e\}} \operatorname{Re} \chi(r)(1-\tilde{\sigma}(r)) d \mu(r), \quad \sigma \in S^{\prime}
$$


Then by (4.1) and (4.8), we see that

$$
q * \sigma-q=\left(\psi^{\prime}-\psi_{\mu}\right) * \sigma-\left(\psi^{\prime}-\psi_{\mu}\right)=\hat{\mu}_{\sigma}-\left(\psi_{\mu} * \sigma-\psi_{\mu}\right)=\mu_{\sigma}(\{e\}) \geq 0
$$

By Lemma 4.3 this implies that $q$ is a nonnegative quadratic form on $\hat{Q}$ and the first requirement is proved.

Secondly, given (4.7), of course $C=\psi(1)$, and $h=\operatorname{Im} \psi$. Denote again $\psi_{\mu}(\chi)=$ $\int_{Q \backslash\{e\}}(1-\operatorname{Re} \chi(r)) d \mu(r)$. By Lemma $4.4 \psi_{\mu}$ is negative definite. By Corollary 2.2

$$
\begin{aligned}
& q(\chi)-\frac{1}{2}\left(R_{\bar{\chi}} q\right)(\chi) \\
= & \lim _{n \rightarrow \infty} \frac{\left(R_{\chi}^{n} q\right)(\chi)}{4 n^{2}} \\
= & \lim _{n \rightarrow \infty} \frac{\left(R_{\chi}^{n} \psi\right)(\chi)}{4 n^{2}}-\lim _{n \rightarrow \infty} \frac{\left(R_{\chi} \psi_{\mu}\right)(\chi)}{4 n^{2}} \\
= & \lim _{n \rightarrow \infty} \frac{\left(R_{\chi}^{n} \psi\right)(\chi)}{4 n^{2}}-\lim _{n \rightarrow \infty} \frac{1}{4 n^{2}} \int\left(1-\operatorname{Re}(\chi(r))^{2 n}\right) d \mu(r)
\end{aligned}
$$

Since $Q$ is locally compact, the Fubini's theorem is available by the inequality (4.2). Obviously

$$
\lim _{n \rightarrow \infty} \frac{1}{4 n^{2}}\left(1-\operatorname{Re}(\chi(r))^{2 n}\right)=0 \quad \text { for each } r \in Q .
$$

If $\chi(r) \neq 0$, let $0<\rho \leq 1$ and $-\pi \leq \theta \leq \pi$ such that $\chi(r)=\rho \exp i \theta$. Then for $n \in N, \frac{\sin n \theta}{n \theta}$ is bounded away from $Q$ on $\left[\frac{\pi}{2}, \pi\right]$, and

$$
\frac{1}{4 n^{2}}(1-\cos 2 n \theta)=\frac{1}{2}\left(\frac{\sin n \theta}{n \theta}\right)^{2}\left(\frac{\theta}{\sin \theta}\right)^{2}\left(\frac{1-\cos 2 \theta}{2}\right) \leq C(1-\cos 2 \theta)
$$

where $C \geq 0$ is a constant.

Also

$$
\frac{1-\rho^{2 n}}{4 n^{2}} \leq \frac{1-\rho}{2 n} \leq \frac{1-\rho^{2}}{2} .
$$

Then

$$
\begin{aligned}
\frac{1}{4 n^{2}}\left(1-\operatorname{Re}(\chi(r))^{2 n}\right) & =\frac{1}{4 n^{2}}\left(1-\rho^{2 n}\right)+\frac{\rho^{2 n}}{4 n^{2}}(1-\cos 2 n \theta) \\
& \leq \frac{1}{2}\left(1-\rho^{2}\right)+\rho^{2 n} C(1-\cos 2 \theta) \\
& \leq \frac{1}{2}\left(1-\rho^{2}\right)+C\left(\rho^{2}-\rho^{2} \cos 2 \theta\right) \\
& \leq \frac{1}{2}\left(1-\rho^{2}\right)+C\left(1-\operatorname{Re}(\chi(r))^{2}\right)
\end{aligned}
$$

Then by the theorem of domainated convergence

$$
\frac{1}{4 n^{2}} \int\left(1-\operatorname{Re}(\chi(r))^{2 n}\right) d \mu(r)=0,
$$


and (4.9) yields

$$
q(\chi)=\lim _{n \rightarrow \infty} \frac{\left(R_{\chi}^{n} \psi\right)(\chi)}{4 n^{2}}+\frac{1}{2}\left(R_{\bar{\chi}} q\right)(\chi) .
$$

By means of $(2.4),\left(R_{\bar{\chi}} q\right)(\chi)=\lim _{n \rightarrow \infty} \frac{\left(R_{\bar{\chi}}^{n} q\right)(\chi)}{2 n}$

$$
\begin{aligned}
\left(R_{\bar{\chi}} q\right)(\chi) & =\lim _{n \rightarrow \infty} \frac{\left(R_{\bar{\chi}}^{n} q\right)(\chi)}{2 n} \\
& =\lim _{n \rightarrow \infty} \frac{\left(R_{\bar{\chi}}^{n} \psi\right)(\chi)}{2 n}-\lim _{n \rightarrow \infty} \frac{1}{2 n} \int_{Q \backslash\{e\}}\left(1-|\chi(r)|^{2 n}\right) d \mu(r)
\end{aligned}
$$

Since

$$
\frac{1}{2 n}\left(1-|\chi(r)|^{2 n}\right) \leq\left(1-|\chi(r)|^{2}\right),
$$

Applying the dominated convergence theorem again, we have

$$
\lim _{n \rightarrow \infty} \frac{1}{2 n} \int_{Q \backslash\{e\}}\left(1-|\chi(r)|^{2 n}\right) d \mu(r)=0,
$$

and

$$
\left(R_{\bar{\chi}} q\right)(\chi)=\lim _{n \rightarrow \infty} \frac{\left(R_{\bar{\chi}}^{n} \psi\right)(\chi)}{2 n}
$$

subistituting in (4.10), the equality (4.7) is stablished.

\section{References}

[1] Ju. M. Berezanskii and A. A. Kalyuzhnyi, Harmonic Analysis in Hypercomplex Systems, Kive, Naukova Dumka(1992).

[2] Ju. M. Berezanskii and A. A. Kalyuzhnyi, Hypercomplex systems and hypergroups: Connections and distributions, Contemporary Mathematics, 183(1995), 21-44.

[3] Ju. M. Berezanskii and Ju. G. Kondratiev, Spectral Methods in Infinite Dimensional Analysis, Kluwer Academic Publishers, Netherlands, 1(1995).

[4] C. Berg, J. P. R. Christensen and P. Ressel, Harmonic Analysis on Semigroups, Springer-Verlage, Berlin-Heidelberg-New York (1984).

[5] C. Berg and G. Forst, Potential Theory on Locally Compact Abelian Groups, SpringerVerlage, Berlin-Heidelberg-New York (1975).

[6] J. Faraut and M. A. Piardello, The Plancherel measure for symmetric groups, Ann. Math. Pure. Appl., 198(1982), 151-155.

[7] K. Harzallah, Sur une. demonstration de la formula de Lévy Khinchine, Ann. Inst. Fourier, 192(1969), 527-532. 
[8] W. Hazod, Über die Lévy Hinčin Formel auf lokalkompakten topologischen Gruppen. Z., Wahrscheinlichkeitstheorie verw. Geb., 25(1973), 301-322.

[9] G. A. Hunt, Semigroups of measures on Lie groups., Trans. Amer. Math. Soc., 81(1956), 264-293.

[10] R. I. Jewett, Spaces with an abstract convolution of measures, Adv. in Math., 18(1975), 1-101.

[11] A. A. Kalyuzhnyi, A theorem on the existence of multiplicative measure, Ukr. Math. Zh., 35(3) (1983), 369-371.

[12] R. Lasser, On the Lévy Hinčin formula for commutative hypergroups, Lecture Notes in Math, 1064(1984), 298-308.

[13] K. R. Parthasarathy, Probability Measures on Metric Spaces, Academic Press, New York, London, (1967).

[14] W. Rudin, Real and Complex Analysis, McGraw-Hill Book Co., New York, (1974).

[15] A. M. Zabal and Buthinah A. Bin Dehaish, Negative definite functions on hypercomplex systems, Accepted for publication in Kyungbook Math. J., 2007. 